\title{
Diffusion and Persistence of Multidrug Resistant Salmonella Typhimurium Strains Phage Type DT120 in Southern Italy
}

\author{
Danila De Vito, ${ }^{1}$ Rosa Monno, ${ }^{1}$ Federica Nuccio, ${ }^{2}$ Marilisa Legretto, ${ }^{2}$ \\ Marta Oliva, ${ }^{2}$ Maria Franca Coscia, ${ }^{1}$ Anna Maria Dionisi, ${ }^{3}$ Carla Calia, ${ }^{1}$ \\ Carmen Capolongo, ${ }^{1}$ and Carlo Pazzani ${ }^{2}$ \\ ${ }^{1}$ Department of Basic Medical Sciences Neurosciences and Sense Organs Medical Faculty, \\ University of Bari Piazza G. Cesare Policlinico, 70124 Bari, Italy \\ ${ }^{2}$ Department of Biology, University of Bari “Aldo Moro", Via E. Orabona 4, 70125 Bari, Italy \\ ${ }^{3}$ Department of Infectious, Parasitic and Immune-Mediated Diseases, National Health Institute, 00161 Rome, Italy \\ Correspondence should be addressed to Carlo Pazzani; carlo.pazzani@uniba.it
}

Received 13 June 2014; Revised 29 August 2014; Accepted 11 September 2014

Academic Editor: Gundlapally S. Reddy

Copyright (C) 2015 Danila De Vito et al. This is an open access article distributed under the Creative Commons Attribution License, which permits unrestricted use, distribution, and reproduction in any medium, provided the original work is properly cited.

\begin{abstract}
Sixty-two multidrug resistant Salmonella enterica serovar Typhimurium strains isolated from 255 clinical strains collected in Southern Italy in 2006-2008 were characterised for antimicrobial resistance genes, pulsotype, and phage type. Most strains (83.9\%) were resistant to ampicillin, chloramphenicol, streptomycin, sulfamethoxazole, and tetracycline (ACSSuT) encoded in $88.5 \%$ by the Salmonella genomic island (SGI1) and in $11.5 \%$ by the InH-like integron (bla $a_{\mathrm{OXA}-30}-$ aadA1) and catA1, sul1, and tet(B) genes. STYMXB.0061 (75\%) and DT120 (84.6\%) were the prevalent pulsotype and phage type identified in these strains, respectively. Five other resistance patterns were found either in single or in a low number of isolates. The pandemic clone DT104 (ACSSuT encoded by SGI1) has been identified in Italy since 1992, while strains DT120 (ACSSuT encoded by SGI1) have never been previously reported in Italy. In Europe, clinical strains DT120 have been reported from sporadic outbreaks linked to the consumption of pork products. However, none of these strains were STYMXB.0061 and SGI1 positive. The prevalent identification and persistence of DT120 isolates would suggest, in Southern Italy, a phage type shifting of the pandemic DT104 clone pulsotype STYMXB.0061. Additionally, these findings raise epidemiological concern about the potential diffusion of these emerging multidrug resistant (SGI linked) DT120 strains.
\end{abstract}

\section{Introduction}

In recent times, diseases caused by foodborne pathogens have been the object of an increasing number of studies [1]. This is owing to the rates of morbidity and mortality that can be achieved by such diseases and the impact that they have in both public health and social economic costs. Among the different bacteria responsible for foodborne diseases, Salmonella enterica represents one of the leading causes of such infections with $S$. Enteritidis and $S$. Typhimurium being the most common serovars isolated from humans in Europe and the USA [2]. However, while in Europe S. Enteritidis still remains the predominant serovar from clinical cases, in Italy it is the serovar $S$. Typhimurium which is prevalent [3].
In the past decades, the emergence and spread of Salmonella multidrug resistant (MDR) strains have also contributed to increasing the impact that these pathogens have had in public health. The insurgence of MDR has generally been linked to acquisition of resistance genes located on plasmids, transposons, genomic islands, and integrons. For example, the multidrug resistance exhibited by the pandemic S. Typhimurium definitive phage type 104, (DT104), is due to resistance genes located within a chromosomal genomic island termed SGI1 composed of a $43 \mathrm{kbp}$ region in which lies a $13 \mathrm{kbp}$ gene cluster of aadA2, floR, tet $(\mathrm{G}), b_{\text {PSE-1 }}$, and sull encoding resistance for streptomycin, chloramphenicol, tetracycline, ampicillin, and sulfamethoxazole, respectively [4]. 
The constant increase of antimicrobial resistance is a global scale problem and it raises concern on the choice of antimicrobials available for the treatment of bacterial infections, particularly for those pathogens of major social relevance. It is thus necessary to implement measures that may contribute to better assessing the extent of resistancegene diffusion among these pathogens. Both monitoring and characterisation of the antimicrobial susceptibility are undoubtedly valuable investigation systems that fulfill the purpose of such measures. Antimicrobial resistance is usually acquired by point mutations in the bacterial genome or by horizontal transfer of genetic elements harbouring resistance genes. Additionally, gene capture and expression elements called integrons have recently been shown to play an important role in acquisition and dissemination of resistance genes. Among the different classes of integrons, class 1 is the most detected and found strongly associated with capture of antimicrobial resistance genes. Class 1 integrons are then recognised as major contributors to the problem of multidrug-resistant pathogens [5]. In S. enterica, class 1 integrons are common and greatly contribute to the extent of antimicrobial resistance in this species [6].

If genetic characterization of antimicrobial resistances was combined with molecular strain typing, it would greatly benefit knowledge on the spread of resistance genes among the clonal groups of Salmonella strains principally identified in the clinical field. Phage typing and pulsed-field gel electrophoresis (PFGE) have become universally recognized as the reference methods for Salmonella strain subtyping.

In Italy, until 2006, the most common phage types identified among MDR clinical $S$. Typhimurium strains were DT104, U302, and DT7 [3]. The first was strongly associated with isolates pulsotype STYMXB.0061 resistant to ampicillin, chloramphenicol, streptomycin, sulfamethoxazole, and tetracycline, while strains U302 and DT7 were pulsotype STYMXB.0079 and resistant to ampicillin, streptomycin, sulfamethoxazole, and tetracycline. However, in Southern Italy (where Salmonella infections are endemic), data on resistance genes and clonal relationship among the MDR strains isolated from clinical cases is poor [7]. In this study, MDR S. Typhimurium strains isolated from several hospitals, from 2006 to 2008, were phage typed and characterised by pulsed field gel electrophoresis (PFGE). The genetic basis of the antimicrobial resistance was also established.

\section{Materials and Methods}

2.1. Bacterial Isolates and Antimicrobial Susceptibility Testing. From January 2006 to December 2008, four hundred and seventy-seven Salmonella strains were isolated from clinical cases which occurred in Southern Italy. Two hundred and fifty-five were S. enterica serovar Typhimurium. Strains were isolated from six different hospitals scattered among the Italian neighbouring regions of Apulia and Basilicata. Biochemical identification of Salmonella was confirmed by an automated system (Microscan, dade Behring, Milan, Italy) and serotyping was performed by agglutination tests with specific antisera $\mathrm{O}$ and $\mathrm{H}$ antigens (Bio-Rad Laboratories, Italy). Classification was performed according to the Kauffmann-White scheme [8].

All isolates were included in the database of the Enternet Surveillance Network at the Institute of Hygiene of the University of Bari (Italy) coordinated by the Istituto Superiore di Sanità, Rome (Italy). There were sixty-two bacteria that were resistant to at least one agent in three or more of the antimicrobial categories tested (namely, aminoglycosides, folate pathway inhibitors, penicillins, phenicols, and tetracyclines) and so defined as MDR [11].

Antimicrobial susceptibility testing was carried out by the agar disk diffusion test on Mueller-Hinton agar (Oxoid, Milan, Italy), following the Clinical and Laboratory Standards Institute (CLSI) guidelines [12]. The antimicrobial disks were ampicillin $(\mathrm{A} ; 10 \mu \mathrm{g})$, chloramphenicol $(\mathrm{C} ; 30 \mu \mathrm{g})$, kanamycin (K; $30 \mu \mathrm{g})$, streptomycin (S; $10 \mu \mathrm{g}$ ), sulfamethoxazole (Su; $25 \mu \mathrm{g}$ ), tetracycline (T; $30 \mu \mathrm{g}$ ), and trimethoprim (Tp $5 \mu \mathrm{g}$ ). E. coli ATCC 25922 was used as a quality control strain.

2.2. PCR Detection of SGI, Class 1 Integrons, and Antimicrobial Resistance Genes. Primers used to detect SGI1, class 1 integrons, and the antimicrobial resistance genes aadAl, $\operatorname{aad} A 2$, and $\operatorname{str} A B$ (encoding resistance for streptomycin; category aminoglycosides), $b l a_{\mathrm{PSE}-1}$ and $b l a_{\mathrm{TEM}}$ (encoding resistance for ampicillin; category penicillins), floR (encoding resistance for chloramphenicol; category phenicols), sul1 and sul2 (encoding resistance for sulfamethoxazole; category folate pathway inhibitors), and $\operatorname{tet}(\mathrm{B})$, tet $(\mathrm{C})$, and $\operatorname{tet}(\mathrm{G})$ (encoding resistance for tetracycline; category tetracyclines) were as reported previously $[9,10]$. Primers to detect the antimicrobial resistance genes $b l a_{\text {OXA-30 }}$ (encoding resistance for ampicillin), catA1 (encoding resistance for chloramphenicol), $d f r A 1$ and $d f r A 12$ (encoding resistance for trimethoprim; category folate pathway inhibitors), and tet(A) and tet(D) (encoding resistance for tetracycline) were designated in this study. All primers are listed in Table 1 . The primers employed in the current study were based on sequences available in GenBank and designed by using the Primer3 (version 4.0.0) software (http://primer3.wi.mit.edu). Genomic DNA was extracted as previously described [13]. PCRs were performed in a total volume of $25 \mu \mathrm{L}$ containing 50 to $100 \mathrm{ng}$ of total DNA, 1X PCR buffer (10 mM Tris- $\mathrm{HCl}, 50 \mathrm{mM} \mathrm{KCl}$, and $1.5 \mathrm{mM} \mathrm{MgCl}$; pH8.3), $200 \mu \mathrm{M}$ of each deoxynucleoside triphosphate (dNTP), $20 \mu \mathrm{M}$ of each primer, and $1 \mathrm{U}$ Taq polymerase (Takara Bio Inc., Otsu, Shiga, Japan).

Antimicrobial gene cassettes integrated into class 1 integrons were amplified with primers 5CS-F and 3CS-R and cloned into pGEM-T Easy vector (Promega, Milan, Italy) in accordance with the manufacturer's instructions. E. coli JM109 was used as a recipient strain. The cloned products were purified using the Pure Yield Plasmid Miniprep System (Promega) and sequenced by the Big Dye Terminator method (BMR Genomics, Padova, Italy). The resulting DNA sequences were analysed for similarity by using the BLAST program available on the NCBI BLAST homepage (http://www.ncbi.nlm.nih.gov/BLAST). 
TABle 1: Primers used for PCR amplification of resistance genes.

\begin{tabular}{|c|c|c|c|c|c|}
\hline Primer & $5^{\prime}-3^{\prime}$ sequence & Gene target & Amplicon size (bp) & TA & Reference \\
\hline aadAl-F & TTTGATCAACGACCTTTTGGAAAC & \multirow{2}{*}{ aadA1 and $a a d A 2$} & \multirow{2}{*}{294} & \multirow{2}{*}{$58^{\circ} \mathrm{C}$} & \multirow{2}{*}[9]{} \\
\hline aadA-R & GGACAACGTAAGCACTACATTTCG & & & & \\
\hline blaoxa30-F & ATTATCTACAGCAGCGCCAGTGCATC & \multirow{2}{*}{$b l a_{\mathrm{OXA}-30}$} & \multirow{2}{*}{716} & \multirow{2}{*}{$63^{\circ} \mathrm{C}$} & \multirow{2}{*}{ This study } \\
\hline blaoxa30-R & TTCGACCCCAAGTTTCCTGTAAGTGC & & & & \\
\hline blaPSE-1-F & GGATTACAATGGCAATCAGCGCTTCC & \multirow{2}{*}{$b l a_{\mathrm{PSE}-1}$} & \multirow{2}{*}{658} & \multirow{2}{*}{$65^{\circ} \mathrm{C}$} & \multirow[b]{2}{*}[10]{} \\
\hline blaPSE-1-R & AATCGCATCATTTCGCTCTGCCATTG & & & & \\
\hline blaTEM1-F & TGAAGATCAGTTGGGTGCACGAGTGG & \multirow{2}{*}{$b l a_{\mathrm{TEM}-1}$} & \multirow{2}{*}{700} & \multirow{2}{*}{$63^{\circ} \mathrm{C}$} & \multirow[b]{2}{*}[9]{} \\
\hline blaTEM1-R & AGTTGCCTGACTCCCCGTCGTGTAGA & & & & \\
\hline catAl-F & CACCGTTGATATATCCCAATGGCATCGT & \multirow{2}{*}{ catA1 } & \multirow{2}{*}{582} & \multirow{2}{*}{$62^{\circ} \mathrm{C}$} & \multirow{2}{*}{ This study } \\
\hline catA1-R & CTGCCGACATGGAAGCCATCACAAAC & & & & \\
\hline dfrAl-F & TATTCCATGGAGTGCCAAAGGTGAAC & \multirow{2}{*}{$d f r A 1$} & \multirow{2}{*}{375} & \multirow{2}{*}{$63^{\circ} \mathrm{C}$} & \multirow{2}{*}{ This study } \\
\hline dfrA1-R & GAGGCGAAGTCTTGGGTAAAAACTGG & & & & \\
\hline dfrA12-F & CAGTACGCATTTATCTCGTTGCTGCG & \multirow{2}{*}{$d f r A 12$} & \multirow{2}{*}{417} & $63^{\circ} \mathrm{C}$ & This study \\
\hline dfrA12-R & CAAGCTCGAATTCTGTTTCGTTGAGC & & & & \\
\hline floR-F & CTCCTTTCGACATCCTCGCTTCACTG & floR & 636 & $64^{\circ} \mathrm{C}$ & \\
\hline floR-R & AGAAGACGAAGAAGGTGCCCATACCG & & & & {$[10]$} \\
\hline sull-F & TCGGCATTCTGAATCTCACCGAGGAC & sull & 786 & $64^{\circ} \mathrm{C}$ & \\
\hline sull-R & AAATTTCGCGAGGGTTTCCGAGAAGG & sul1 & 700 & $04 \mathrm{C}$ & [10] \\
\hline sul2-F & GACAGTTTCTCCGATGGAGGCCGGTA & sul2 & 700 & $64^{\circ} \mathrm{C}$ & \\
\hline sul2-R & GTGTGCGGATGAAGTCAGCTCCACCT & & 100 & & [10] \\
\hline strA-F & TGGTTGCCTGTCAGAGGCGGAGAATC & $\operatorname{str} A B$ & 1466 & $63^{\circ} \mathrm{C}$ & [Q] \\
\hline strB-R & ATCGACGTCCAGCGCACGAGAGAATG & & & & [9] \\
\hline tetA-F & CCTGATCGTAATTCTGAGCACTGTCG & tet(A) & 1089 & $63^{\circ} \mathrm{C}$ & This study \\
\hline tetA-R & CACCCGTTCCACGTTGTTATAGAAGC & & & & \\
\hline tetB-F & CAGGTTATCTTTGCTCCTTGGCTTGG & $\operatorname{tet}(\mathrm{B})$ & 1014 & $63^{\circ} \mathrm{C}$ & [0] \\
\hline tetB-R & TTGAGGGGTTAACATGAAGGTCATCG & & & & [9] \\
\hline tetC-F & GGATATCGTCCATTCCGACAGCATCG & tet $(\mathrm{C})$ & 745 & $63^{\circ} \mathrm{C}$ & [9] \\
\hline tetC-R & GATAATGGCCTGCTTCTCGCCGAAAC & & & & [9] \\
\hline tetD-F & ACTGTCCAATGTGCTGTGGATGTTGT & tet $(D)$ & 747 & $63^{\circ} \mathrm{C}$ & This study \\
\hline tetD-R & CTGACCAGCACACCCTGTAGTTTTCC & (c) & 17 & & \\
\hline tetG-F & GAGCCGCAGTCGATTACACGATTATG & $\operatorname{tet}(\mathrm{G})$ & 680 & $64^{\circ} \mathrm{C}$ & \\
\hline tetG-R & CAACAGAATCGGGAACACCATCCATC & & & & [10] \\
\hline intI1-F & CGAACCGAACAGGCTTATGTCCACTG & intI1 & 838 & $63^{\circ} \mathrm{C}$ & [10] \\
\hline intI1-R & CATCGTCGTAGAGACGTCGGAATGG & & & & {$[10]$} \\
\hline $5 \mathrm{CS}-\mathrm{F}$ & GCCTCGGGCATCCAAGCAGCAAGC & $5^{\prime} \mathrm{CS}$ attI 1 end $3^{\prime} \mathrm{CS}$ & variable & $65^{\circ} \mathrm{C}$ & This study \\
\hline 3CS-R & CTTGACCTGATAGTTTGGCTGTGAGCAA & & & & \\
\hline U7-L12 & ACACCTTGAGCAGGGCAAAG & $\operatorname{tdhF}$ & 500 & $60^{\circ} \mathrm{C}$ & [4] \\
\hline LJ-R1 & AGTTCTAAAGGTTCGTAGTCG & int & & & [4] \\
\hline 104-RJ & TGACGAGCTGAAGCGAATTG & S044 & 515 & $60^{\circ} \mathrm{C}$ & {$[4]$} \\
\hline C9-L & AGCAAGTGTGCGTAATTTGG & int2 & & & [4] \\
\hline
\end{tabular}

2.3. PFGE and Phage Typing. Genomic restriction was performed according to the standardized PulseNet Salmonella protocol [14]. Agarose-embedded DNA was digested with $40 \mathrm{U}$ of $\mathrm{XbaI}$ for $3 \mathrm{~h}$ at $37^{\circ} \mathrm{C}$. The restriction fragments were separated by electrophoresis in Tris-borate-EDTA $(44.5 \mathrm{mM}$ Tris-borate, $1 \mathrm{mM}$ EDTA; $\mathrm{pH} 8.0$ ) at $14^{\circ} \mathrm{C}$ for $20 \mathrm{~h}$ using a CHEF-DR III (Bio-Rad, Milan, Italy). Electrophoresis conditions were as follows: $6 \mathrm{~V} / \mathrm{cm}$, angle of $120^{\circ} \mathrm{C}$, for $20 \mathrm{~h}$ with pulse times of 2.2 to 63.8 s. The Salmonella Braenderup H9812 strain was used as a molecular standard. The PFGE agarose gels were stained with ethidium bromide $(40 \mu \mathrm{g} / \mathrm{mL})$ and the DNA band images were acquired by the Gel Doc-It photo documentation system (Gel Doc-It photo documentation system, UVP, Upland, CA, USA).

PFGE profiles in digital tagged image file format (TIFF files) were analyzed with the BioNumerics software package v.6.6 (Applied Maths, Sint-Martens-Latem, Belgium) [15]. DNA profiles differing in one or more DNA fragments were considered as distinct patterns. Strains with a coefficient of similarity $\geq 90 \%$ were classified as genetically closely related. 


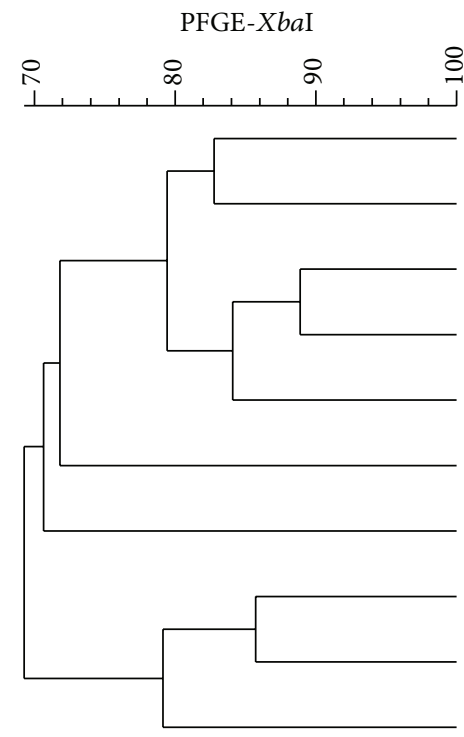

PFGE-XbaI

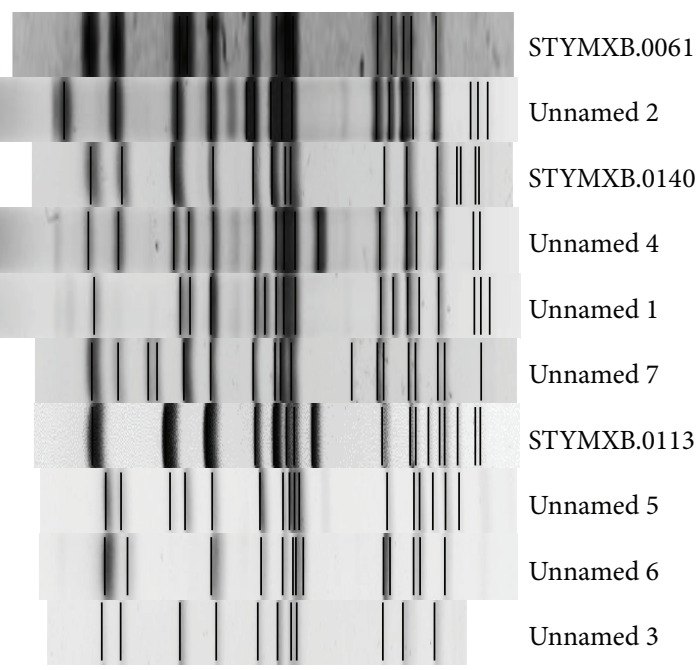

FIGURE 1: PFGE profiles and cluster analysis of Salmonella enterica serotype typhimurium strains analysed in this study. All representative variants of single PFGE patterns are included.

All PFGE profiles were compared with those included in the PulseNet-Europe international database and named with a six letter code followed by a four digit numerical identifier, for example, STYMXB.0006. Dendrogram and cluster analysis were performed using algorithms available within the BioNumerics software package v.6.6 (Figure 1). Percent similarity between different chromosomal fingerprints was scored by the Dice coefficient. The unweighted pair group method with arithmetic means (UPGMA), with a $1.00 \%$ tolerance limit and $1.00 \%$ optimisation, was used to obtain the dendrogram. DNA profiles differing by one or more DNA fragments were considered as distinct patterns. Phage typing was performed according to the standard procedure [16].

\section{Results}

3.1. Antimicrobial Susceptibility, Pulsotype, and Phage Typing. Six distinct resistance patterns were identified within the $62 \mathrm{MDR} S$. typhimurium strains analysed in this study (Table 2). The resistance pattern to ampicillin, chloramphenicol, streptomycin, sulfamethoxazole, tetracycline, and trimethoprim (termed resistance pattern ACSSuT) was that prevalent $(83.9 \%)$, being identified in 52 strains. All others resistance patterns were identified either in single (ASSuT, ASSuTp, and AKSSuT) or in a low number of isolates (ASuTTp and ACSSuTTp).

Strains were characterised by genomic $\mathrm{XbaI}$ restriction patterns and resolved through a pulsed field gel electrophoresis and phage typing. PFGE profiles were compared with those included in the PulseNet-Europe international database and specific pulsotypes were assigned when the coefficient of similarity had a value $\geq 90 \%$. Among the 52 strains with resistance pattern ACSSuT, 39 were pulsotype STYMXB.0061 with phage types distributed as follows: DT120 (84.6\%), U302 (13.5\%), and RDNC (1.9\%). The remaining 13 strains with resistance pattern ACSSuT were pulsotypes STYMXB.0140 (4) or unnamed (9), and all of them were classified as phage type DT120. Five strains resistance pattern ASuTTp were pulsotype STYMXB.0113: two strains were classified DT35 and three DT193. Two isolates exhibiting the resistance pattern ACSSuTTp and the single isolates with resistance patterns ASSuT, ASSuTp, and AKSSuT were pulsotype unnamed and phage types DT120, DT7var, DT1, and U302, respectively.

3.2. Resistance Genes Associated with SGI1. Identification of SGI1 and associated resistance genes was performed by PCR. Among the 52 strains with resistance pattern ACSSuT, 46 were positive for the presence of SGI1. Resistance genes ( aadA2, floR, tet $(\mathrm{G}), b l a_{\mathrm{PSE}-1}$, and sull) usually harboured by SGI1 were identified and their genetic organization proved indistinguishable from that reported for SGI1 [4]. SGI1 and associated resistance genes were also identified in strains with resistance pattern ACSSuTTp and found identical to that identified in strains with resistance pattern ACSSuT. Strains with resistance patterns ASuTTp, AKSSuT, ASSuT, and ASSuTp were all negative for the presence of SGI1.

3.3. Resistance Genes and Class 1 Integrons. Class 1 integrons were identified in all strains with resistance patterns ACSSuT and ACSSuTTp. Two amplicons of 1,009 bp and 1,197 bp were obtained among the 46 strains with resistance pattern ACSSuT and SGI1 positive. The nucleotide sequence of the gene cassettes was determined and two open reading frames (ORFs) of 852 and 987 bp were detected and found homologous to aadA2 and $b l a_{\mathrm{PSE}-1}$, respectively. PCR analysis was further extended for detection of sull and its linkage to class 1 integron. The sull gene was found associated only with the class 1 integron harbouring the $b l a_{\mathrm{PSE}-1}$ gene cassette. These data further confirmed the presence of SGI1. An amplicon of 2,013 bp was obtained from the remaining 6 strains with 
TABle 2: Antimicrobial susceptibility, phage types, pulsotypes, PCR detection of SGIl, class 1 integrons, and resistance genes in $S$. Typhimurium strains isolated in Southern Italy in 2006-2008.

\begin{tabular}{|c|c|c|c|c|c|c|c|c|}
\hline \multirow{2}{*}{$\begin{array}{l}\text { PulseNet Europe } \\
\text { nomenclature }\end{array}$} & \multirow{2}{*}{$\begin{array}{l}\text { Phage type } \\
\text { (no. of } \\
\text { strains) }\end{array}$} & \multirow{2}{*}{$\begin{array}{c}\text { Year } \\
\text { (no. of } \\
\text { strains) }\end{array}$} & \multirow{2}{*}{$\begin{array}{l}\text { Resistance } \\
\text { pattern }^{\mathrm{a}}\end{array}$} & \multicolumn{2}{|r|}{$\begin{array}{c}\text { Class } 1 \\
\text { integrons }\end{array}$} & \multirow[b]{2}{*}{$\begin{array}{l}\text { Resistance } \\
\text { gene(s) }\end{array}$} & \multicolumn{2}{|c|}{ SGI1 } \\
\hline & & & & intI1 & $\begin{array}{c}\text { Gene } \\
\text { cassette(s) }\end{array}$ & & $\begin{array}{c}\text { Left } \\
\text { junction }\end{array}$ & $\begin{array}{c}\text { Right } \\
\text { junction }\end{array}$ \\
\hline \multirow{4}{*}{ STYMXB.0061 } & DT120 (31) & $\begin{array}{c}2006(9) ; \\
2007(13) ; \\
2008(9)\end{array}$ & ACSSuT & + & aadA2; $b l a_{\mathrm{PSE}-1}$ & floR; sull; tet $(G)$ & + & + \\
\hline & \multirow{2}{*}{ U302 (7) } & \multirow{2}{*}{$\begin{array}{l}2006(1) \\
2008(4) \\
2008(2)\end{array}$} & & + & " & & + & + \\
\hline & & & " & + & $\prime \prime$ & " & + & - \\
\hline & RDNC (1) & $2006(1)$ & " & + & "I & " & + & + \\
\hline \multirow[t]{2}{*}{ STYMXB.0113 } & ST193 (3) & 2006 & ASuTTp & - & - & $\begin{array}{l}\text { bla } \\
\operatorname{tet}(A) ; d f r A 12\end{array}$ & - & - \\
\hline & DT35 $(2)^{\mathrm{b}}$ & " & " & - & - & $\begin{array}{l}b l a_{\mathrm{TEM}} ; s u l 2 ; \\
\operatorname{tet}(A)\end{array}$ & - & - \\
\hline STYMXB.0140 & DT120 (4) & $2007(4)$ & ACSSuT & + & $b l a_{\mathrm{OXA}-30}-a a d A 1$ & $\begin{array}{l}\text { catAl; sull; } \\
\text { tet }(B)\end{array}$ & - & - \\
\hline Unnamed 1 & DT120 (9) & $\begin{array}{l}2006(2) ; \\
2007(3)\end{array}$ & ACSSuT & + & $a a d A 2 ; b l a_{\mathrm{PSE}-1}$ & floR; sull; tet $(G)$ & + & + \\
\hline Unnamed 2 & " & $2008(2)$ & $\prime \prime$ & + & $\prime \prime$ & " & + & + \\
\hline Unnamed 3 & " & $2008(2)$ & $\prime \prime$ & + & $b l a_{\mathrm{OXA}-30}-a a d A 1$ & $\begin{array}{l}\text { catAl; sull; } \\
\quad \text { tet }(B)\end{array}$ & - & - \\
\hline Unnamed 4 & " & $2007(2)$ & ACSSuTTp & + & $\begin{array}{c}a a d A 2 ; b l a_{\mathrm{PSE}-1} \\
d f r A 1-a a d A 1\end{array}$ & floR; sull; tet $(G)$ & + & + \\
\hline Unnamed 5 & DT7var ${ }^{\mathrm{c}}$ & 2007 (1) & AKSSuT & - & J & $\begin{array}{l}b l a_{\mathrm{TEM}} ; \operatorname{sul} 2 ; \\
\operatorname{str} A B ; \operatorname{tet}(B)\end{array}$ & - & - \\
\hline Unnamed 6 & DT1 & $2008(1)$ & ASSuT & - & - & $\begin{array}{l}\text { bla } a_{\mathrm{TEM}} ; \text { sul } 2 ; \\
\operatorname{str} A B ; \operatorname{tet}(A)\end{array}$ & - & - \\
\hline Unnamed 7 & $\mathrm{U} 302^{\mathrm{d}}$ & 2008 (1) & ASSuTp & - & - & $\begin{array}{l}b^{b l a_{\mathrm{TEM}}} ; \text { sul2; } \\
\quad d f r A 12\end{array}$ & - & - \\
\hline
\end{tabular}

${ }^{a} \mathrm{~A}$, ampicillin; C, chloramphenicol; K, kanamycin; S streptomycin; Su, sulfamethoxazole; T, tetracycline; and Tp, trimethoprim.

${ }^{\mathrm{b}}$ The resistance to Tp was not identified.

${ }^{c}$ The resistance to $\mathrm{K}$ was not identified.

${ }^{\mathrm{d}}$ The resistance to $S$ and $T p$ was not identified.

RDNC: the reaction pattern is not conforming to a recognised phage type.

Key: +, positive; -, negative.

resistance pattern ACSSuT (SGI1 negative). Two ORFs of 813 and $987 \mathrm{bp}$, organised as a gene cassette array, were identified and found homologous to $b l a_{\mathrm{OXA}-30}$ and aadA1. The presence of both $b l a_{\mathrm{OXA}-30}$ and aadAl, in these 6 isolates, was also confirmed by PCR and sull was found associated with the gene cassette array. Detection of catAl and tet(B) completed the analysis of the resistance genes in these 6 isolates.

The strains with resistance pattern ACSSuTTp additional to SGIl, indistinguishable from that identified in strains with resistance pattern ACSSuT, harboured another class1 integron element. The gene cassette amplicon (1,534 bp) was sequenced and two ORFs of 474 and $789 \mathrm{bp}$ were identified. The ORFs were found homologous to dfrA1 and aadAl, respectively.

In strains with resistance pattern ASuTTp, the identified resistance genes were $b l a_{\mathrm{TEM}}$, sul2, tet(B), and $d f r A 12$. In the single strains with resistance patterns AKSSuT, ASSuT, and ASSuTp, the resistance genes were $b l a_{\mathrm{TEM}}, \operatorname{sul} 2, \operatorname{str} A B$, tet(B); bla $a_{\mathrm{TEM}}, \operatorname{sul} 2, \operatorname{str} A B$, tet(A); and bla $a_{\mathrm{TEM}}, s u l 2, d f r A 12$, respectively. The genetic basis for resistances to kanamycin (resistance pattern AKSSuT) and streptomycin (resistance pattern ASSuTp) was not identified.

\section{Discussion}

Salmonella infections still remain a major public health concern in many countries with an estimated incidence of 15.1 cases per 100,000 persons in the United States and 42.2 cases per 100,000 persons in the EU [17]. In Italy, the average annual incidence is 14 cases per 100,000 persons [18]. However, since Salmonella infections are generally based on the identification of Salmonella strains from stool culture, data officially reported are likely to represent only a fraction of infections which really occur. The difference between reported cases and those probably occurring has recently been investigated by a study that assessed the seroincidence of Salmonella infections in eight European countries, Italy included [18]. The study demonstrated the misleading data 
on the incidence of Salmonella infections based only on numbers of stool culture-confirmed cases. Hence, the need to integrate epidemiological data with those gained by novel methods of investigation such as the assessment of cases by seroincidence.

Salmonella is also the leading cause of hospitalization from foodborne pathogens and the increase of antimicrobial resistance often represents a problem for the choice of a proper antimicrobial therapy (if required). A clear example is provided by the insurgence in the mid-1980s (in the United Kingdom) of the MDR $S$. Typhimurium phage type 104, resistance pattern ACSSuT encoded by SGI1, and its subsequent diffusion. Shortly after its emergence, detection of the MDR S. Typhimurium DT140 constantly increased among 29 countries which took part in a survey conducted from 1992 to 2001 [19]. In the following years, this clone became virtually pandemic.

In Italy, a survey conducted from 1992 to 2000 highlighted the presence of this pandemic clone from 1992 [20]. Detection of the MDR S. Typhimurium DT104, resistance pattern ACSSuT, remained constant from 2002 to 2004 accounting for $20 \%$ of the S. Typhimurium strains of clinical origin [21].

In this study, we report the characterisation of $62 \mathrm{MDR}$ $S$. Typhimurium strains isolated from clinical cases which occurred in Southern Italy from 2004 to 2006. The incidence of S. Typhimurium was of 53.5\% (255 strains out of the 477), data that confirmed the prevalent detection of this serovar in Italy. Multidrug resistance was detected in $24.3 \%$ of the S. Typhimurium strains. STYMXB.0061 was the prevalent (62.9\%) identified pulsotype. This datum is in agreement with that reported by other studies STYMXB.0061 being one of the most common profiles identified in S. Typhimurium [22] and in Italy reported as the second PFGE profile detected in human isolates from 2003 to 2006 [23]. STYMXB.0061 is strongly associated with the phage type 104 and this relationship is documented by a number of studies on $S$. Typhimurium strains isolated from both human and animal sources [10, 22, 23]. SGI1, first detected in S. Typhimurium strains phage type 104, has also been identified in strains with phage types related to DT104 such as DT120, U302, or DT12 [24]. Strains DT120 or DT12 with resistance pattern ACSSuT exhibited PFGE profiles identical to those shown by isolates DT104 with resistance pattern ACSSuT and distinct from profiles exhibited by strains of the same phage types [25]. Such a feature has been supposed due to a change in phage susceptibility in a small proportion of $S$. Typhimurium strains phage type 104 with resistance pattern ACSSuT rather than to horizontal gene transfer of resistance determinants. Additionally, phage typing has widely been used for strain characterisation, and in surveillance and outbreaks investigation [26]. Interestingly, in our survey, none of the isolates STYMXB.0061 with resistance pattern ACSSuT was DT104. Thirty-one (79.5\%) were phage type DT120 and seven (15.2\%) phage type U302. The prevalent identification of phage type DT120 would suggest, at least in Southern Italy, a phage type shifting of the S. Typhimurium strain DT104 (resistance pattern ACSSuT, pulsotype STYMXB.0061) of clinical origin. This possibility is also supported by the virtual absence of phage type DT120 detection in S. Typhimurium strains of human and non-human sources isolated in Italy at least until 2000 [27, 28]. In a subsequent study performed in Italy (2002-2004) on S. Typhimurium strains isolated from human and animal sources, DT120 was rarely detected among the 367 identified MDR strains (resistance pattern ACSSuT). Only 7 isolates were DT120 while 196 (72.3\%) were DT104. Additionally, for all of these 7 strains, neither source of isolation nor antimicrobial resistance genes were specified [3].

MDR S. Typhimurium DT120 strains (e.g. resistant to ASSuT, encoded by $b l a_{\mathrm{TEM}}, \operatorname{str} A B, \operatorname{sul} 2$ and tet(B)) have recently emerged in European countries, with pigs being the likely reservoir of infection [29]. Indeed, in a survey on the prevalence of Salmonella in slaughtered pigs, conducted in the EU from 2006 to 2007, the serovars Typhimurium and Derby were those most frequently identified [30].

The potential impact of S. Typhimurium DT120 in public health has recently been highlighted by clinical outbreaks which occurred in Denmark in 2008 and the UK in 2011. Infections linked to the consumption of pork or pork products [31-34]. Moreover, in Greece, DT120 is associated with the most frequently occurring clones in human and pigs (2006-2011) [33]. However, none of these DT120 isolates was STYMXB.0061 (STYMXB.0083 was the pulsotype detected from the outbreak in UK, while STYMXB.0010 was that identified in Denmark and Greece) and, above all, the antimicrobial resistance patterns (mainly ASSuT and ACSSuTTp) were not linked to the presence of SGI1.

In Italy, the phage type DT120 has commonly been detected from finishing pigs in slaughterhouses [35]. However, these data come from slaughterhouses located in Northern Italy while data from Southern Italy, to the best of our knowledge, are still unavailable. Additionally, none of the $S$. Typhimurium DT120 strains isolated from slaughterhouses in Northern Italy was resistance pattern ACSSuT and no data was available on their pulsotype. It would be interesting to investigate the molecular and phenotypic features of $S$. Typhimurium strains isolated from swine samples in Southern Italy. This might help determine possible epidemiological routes that have contributed to the spread of $S$. Typhimurium strains phage type DT120 that we detected from the clinical isolates.

The integrons $d f r A 1-a a d A 1$ and $b l a_{\text {OXA-30-aadA1 have }}$ been identified in $S$. Typhimurium strains isolated from different geographic areas [6]. The identification of distinct groups of integrons together with phage typing and molecular strain characterisation represents valuable markers to assess possible clonal expansion. Interestingly, the above types of integrons were all identified in MDR strains DT120 with different pulsotype (STYMXB.0113 or Unnamed) highlighting the presence of distinct DT120 clones among clinical cases in Southern Italy.

\section{Conclusions}

In this study, we first described the extent of the antimicrobial resistance genes and associated genetic elements among clinical MDR S. Typhimurium strains isolated from an Italian area where Salmonella infections are endemic. Data 
presented suggest, in this endemic area, a phage type shifting of the pandemic clone DT104 (resistance pattern ACSSuT) to DT120. Such a shifting, to the best of our knowledge, has occurred recently since no clinical $S$. Typhimurium strains DT120 (resistance pattern ACSSuT encoded by SGI encoded) was not reported until 2004. The extent and persistence of these findings raise concerns in public health about the possible diffusion of these emerging DT120 clones.

\section{Conflict of Interests}

The authors declare that they have no conflict of interests.

\section{References}

[1] X. Zhao, C.-W. Lin, J. Wang, and D. H. Oh, "Advances in rapid detection methods for foodborne pathogens," Journal of Microbiology and Biotechnology, vol. 24, no. 3, pp. 297-312, 2014.

[2] F.-X. Weill, F. Guesnier, V. Guibert et al., "Multidrug resistance in Salmonella enterica serotype typhimurium from humans in France (1993 to 2003)," Journal of Clinical Microbiology, vol. 44, no. 3, pp. 700-708, 2006.

[3] C. Graziani, L. Busani, A. M. Dionisi et al., "Antimicrobial resistance in Salmonella enterica serovar Typhimurium from human and animal sources in Italy, Veterinary Microbiology, vol. 128, no. 3-4, pp. 414-418, 2008.

[4] D. Boyd, G. A. Peters, A. Cloeckaert et al., "Complete nucleotide sequence of a 43-kilobase genomic island associated with the multidrug resistance region of Salmonella enterica serovar typhimurium DT104 and its identification in phage type DT120 and serovar agona," Journal of Bacteriology, vol. 183, no. 19, pp. 5725-5732, 2001

[5] R. M. Hall and C. M. Collis, "Antibiotic resistance in gramnegative bacteria: the role of gene cassettes and integrons," Drug Resistance Updates, vol. 1, no. 2, pp. 109-119, 1998.

[6] M. G. Krauland, J. W. Marsh, D. L. Paterson, and L. H. Harrison, "Integron-mediated multidrug resistance in a global collection of nontyphoidal Salmonella enterica isolates," Emerging Infectious Diseases, vol. 15, no. 3, pp. 388-396, 2009.

[7] R. Monno, C. Rizzo, D. de Vito et al., "Prevalence, antimicrobial resistance, and extended-spectrum $\beta$-lactamases characterization of Salmonella isolates in Apulia, southern Italy (20012005)," Microbial Drug Resistance, vol. 13, no. 2, pp. 124-129, 2007.

[8] M. Y. Popoff, J. Bockemühl, and L. L. Gheesling, "Supplement 2002 (no. 46) to the Kauffmann-White scheme," Research in Microbiology, vol. 155, no. 7, pp. 568-570, 2004.

[9] A. Camarda, N. Pugliese, A. Pupillo et al., "Resistance genes, phage types and pulsed field gel electrophoresis pulsotypes in Salmonella enterica strains from laying hen farms in southern Italy," International Journal of Environmental Research and Public Health, vol. 10, no. 8, pp. 3347-3362, 2013.

[10] A. Camarda, A. Pupillo, N. Pugliese et al., "Phenotypic and genetic traits of Salmonella enterica subsp. serovar Typhimurium strains causing salmonellosis foci in rabbit farms from Southern Italy in 1999-2003," Research in Veterinary Science, vol. 94, no. 3, pp. 394-398, 2013.

[11] A.-P. Magiorakos, A. Srinivasan, R. B. Carey et al., "Multidrugresistant, extensively drug-resistant and pandrug-resistant bacteria: an international expert proposal for interim standard definitions for acquired resistance," Clinical Microbiology and Infection, vol. 18, no. 3, pp. 268-281, 2012.

[12] Clinical and Laboratory Standards Institute, Performance Standard for Antimicrobial Disk Susceptibility Tests; Approved Standard M2-A9, CLSI, Wayne, Pa, USA, 9th edition, 2006.

[13] N. Pugliese, F. Maimone, M. Scrascia, S. F. Materu, and C. Pazzani, "SXT-related integrating conjugative element and IncC plasmids in Vibrio cholerae O1 strains in Eastern Africa," The Journal of Antimicrobial Chemotherapy, vol. 63, no. 3, pp. 438442, 2009.

[14] E. M. Ribot, M. A. Fair, R. Gautom et al., "Standardization of pulsed-field gel electrophoresis protocols for the subtyping of Escherichia coli O157:H7, Salmonella, and Shigella for PulseNet," Foodborne Pathogens and Disease, vol. 3, no. 1, pp. 59-67, 2006.

[15] L. Busani, C. Graziani, A. Battisti et al., "Antibiotic resistance in Salmonella enterica serotypes Typhimurium, Enteritidis and Infantis from human infections, foodstuffs and farm animals in Italy," Epidemiology and Infection, vol. 132, no. 2, pp. 245-251, 2004.

[16] L. R. Ward, J. D. de Sa, and B. Rowe, "A phage-typing scheme for Salmonella enteritidis," Epidemiology and Infection, vol. 99, no. 2, pp. 291-294, 1987.

[17] S. D. Alcaine, L. D. Warnick, and M. Wiedmann, "Antimicrobial resistance in nontyphoidal Salmonella," Journal of Food Protection, vol. 70, no. 3, pp. 780-790, 2007.

[18] G. Falkenhorst, J. Simonsen, T. H. Ceper et al., "Serological cross-sectional studies on salmonella incidence in eight European countries: no correlation with incidence of reported cases," BMC Public Health, vol. 12, no. 1, article 523, 2012.

[19] M. Helms, S. Ethelberg, and K. Molbak, "International Salmonella Typhimurium DT104 infections, 1992-2001," Emerging Infectious Diseases, vol. 11, pp. 859-867, 2005.

[20] A. Carattoli, E. Filetici, L. Villa, A. M. Dionisi, A. Ricci, and I. Luzzi, "Antibiotic resistance genes and Salmonella genomic island 1 in Salmonella enterica serovar Typhimurium isolated in Italy," Antimicrobial Agents and Chemotherapy, vol. 46, no. 9, pp. 2821-2828, 2002.

[21] A. Cawthorne, P. Galetta, M. Massari, A. M. Dionisi, E. Filetici, and I. Luzzi, "Salmonella typhimurium DT104, Italy," Emerging Infectious Diseases, vol. 12, no. 8, article 1289, 2006.

[22] A. J. Gatto, T. M. Peters, J. Green et al., "Distribution of molecular subtypes within Salmonella enterica serotype Enteritidis phage type 4 and S. Typhimurium definitive phage type 104 in nine European countries, 2000-2004: results of an international multi-centre study," Epidemiology and Infection, vol. 134, no. 4, pp. 729-736, 2006.

[23] A. M. Dionisi, C. Graziani, C. Lucarelli et al., "Molecular characterization of multidrug-resistant strains of salmonella enterica serotype typhimurium and monophasic variant (S. 4,[5],12:i:-) isolated from human infections in Italy," Foodborne Pathogens and Disease, vol. 6, no. 6, pp. 711-717, 2009.

[24] H. Targant, C. Ponsin, C. Brunet et al., "Characterization of resistance genes in multidrug-resistant Salmonella enterica serotype typhimurium isolated from diseased cattle in France (2002 to 2007)," Foodborne Pathogens and Disease, vol. 7, no. 4, pp. 419-425, 2010.

[25] A. J. Lawson, M. U. Dassama, L. R. Ward, and E. John Threlfall, "Multiply Resistant (MR) Salmonella enterica serotype Typhimurium DT 12 and DT 120: A case of MR DT 104 in disguise?" Emerging Infectious Diseases, vol. 8, no. 4, pp. 434436, 2002. 
[26] L. Majtanova, J. Majtan, and V. Majtan, “Trends in phage types of Salmonella enterica serovars Enteritidis and Typhimurium isolated in Slovakia from 1995 to 2009," Diagnostic Microbiology and Infectious Disease, vol. 69, no. 4, pp. 454-456, 2011.

[27] M. Fantasia, E. Filetici, S. Arena, and S. Mariotti, "Serotype and phage type distribution of salmonellas from human and nonhuman sources in Italy in the period 1973-1995," European Journal of Epidemiology, vol. 14, no. 7, pp. 701-710, 1998.

[28] F. Pasquali, A. De Cesare, A. Ricci, C. Kehrenberg, S. Schwarz, and G. Manfreda, "Phage types, ribotypes and tetracycline resistance genes of Salmonella enterica subsp. enterica serovar Typhimurium strains isolated from different origins in Italy," Veterinary Microbiology, vol. 103, no. 1-2, pp. 71-76, 2004.

[29] K. L. Hopkins, M. Kirchner, B. Guerra et al., "Multiresistant Salmonella enterica serovar 4,[5],12:i:- in Europe: a new pandemic strain?” Eurosurveillance, vol. 15, no. 22, 2010.

[30] EFSA (European Food Safety Authority), "Report of the Task Force on Zoonoses Data Collection on the analysis of the baseline survey on the prevalence of Salmonella in slaughter pigs, Part A," The EFSA Journal, vol. 135, no. 5, pp. 1-111, 2008.

[31] S. Ethelberg, A. Wingstrand, T. Jensen et al., "Large outbreaks of Salmonella typhimurium infection in Denmark in 2008," Eurosurveillance, vol. 13, no. 44, article 19023, 2008.

[32] E. L. Best, M. D. Hampton, S. Ethelberg, E. Liebana, F. A. Clifton-Hadley, and E. J. Threlfall, "Drug-resistant Salmonella Typhimurium DT 120: use of PFGE and MLVA in a putative international outbreak investigation," Microbial Drug Resistance, vol. 15, no. 2, pp. 133-138, 2009.

[33] G. Mandilara, M. Lambiri, M. Polemis, M. Passiotou, and A. Vatopoulos, "Phenotypic and molecular characterisation of multiresistant monophasic Salmonella typhimurium (1,4,[5],12:I:-) in Greece, 2006 to 2011," Eurosurveillance, vol. 18, no. $22,2013$.

[34] K. Paranthaman, S. Haroon, S. Latif et al., "Emergence of a multidrug-resistant (ASSuTTm) strain of Salmonella enterica serovar typhimurium DT120 in England in 2011 and the use of multiple-locus variable-number tandem-repeat analysis in supporting outbreak investigations," Foodborne Pathogens and Disease, vol. 10, no. 10, pp. 850-855, 2013.

[35] S. Bonardi, L. Bassi, F. Brindani et al., "Prevalence, characterization and antimicrobial susceptibility of Salmonella enterica and Yersinia enterocolitica in pigs at slaughter in Italy," International Journal of Food Microbiology, vol. 163, no. 2-3, pp. 248-257, 2013. 

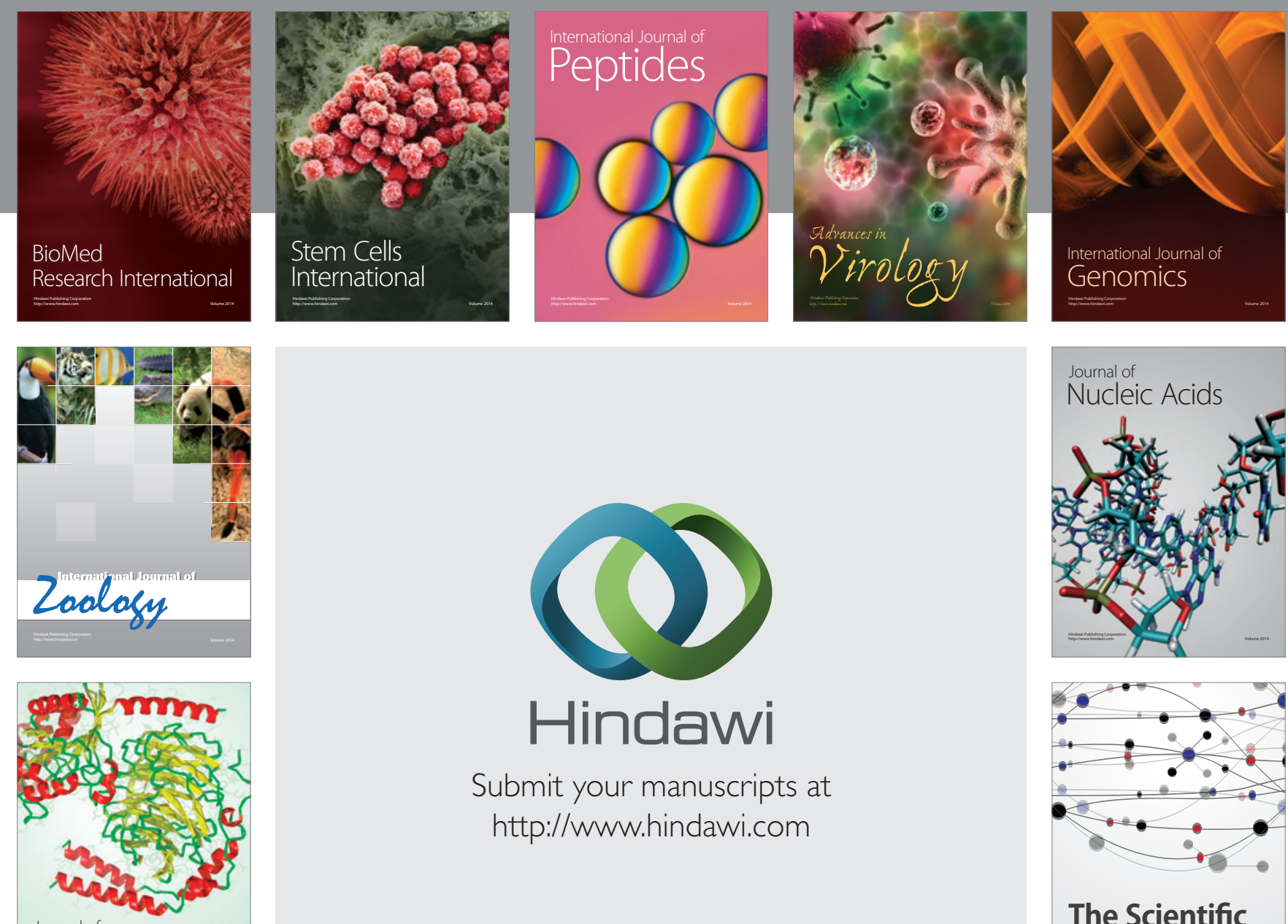

Submit your manuscripts at

http://www.hindawi.com

Journal of
Signal Transduction
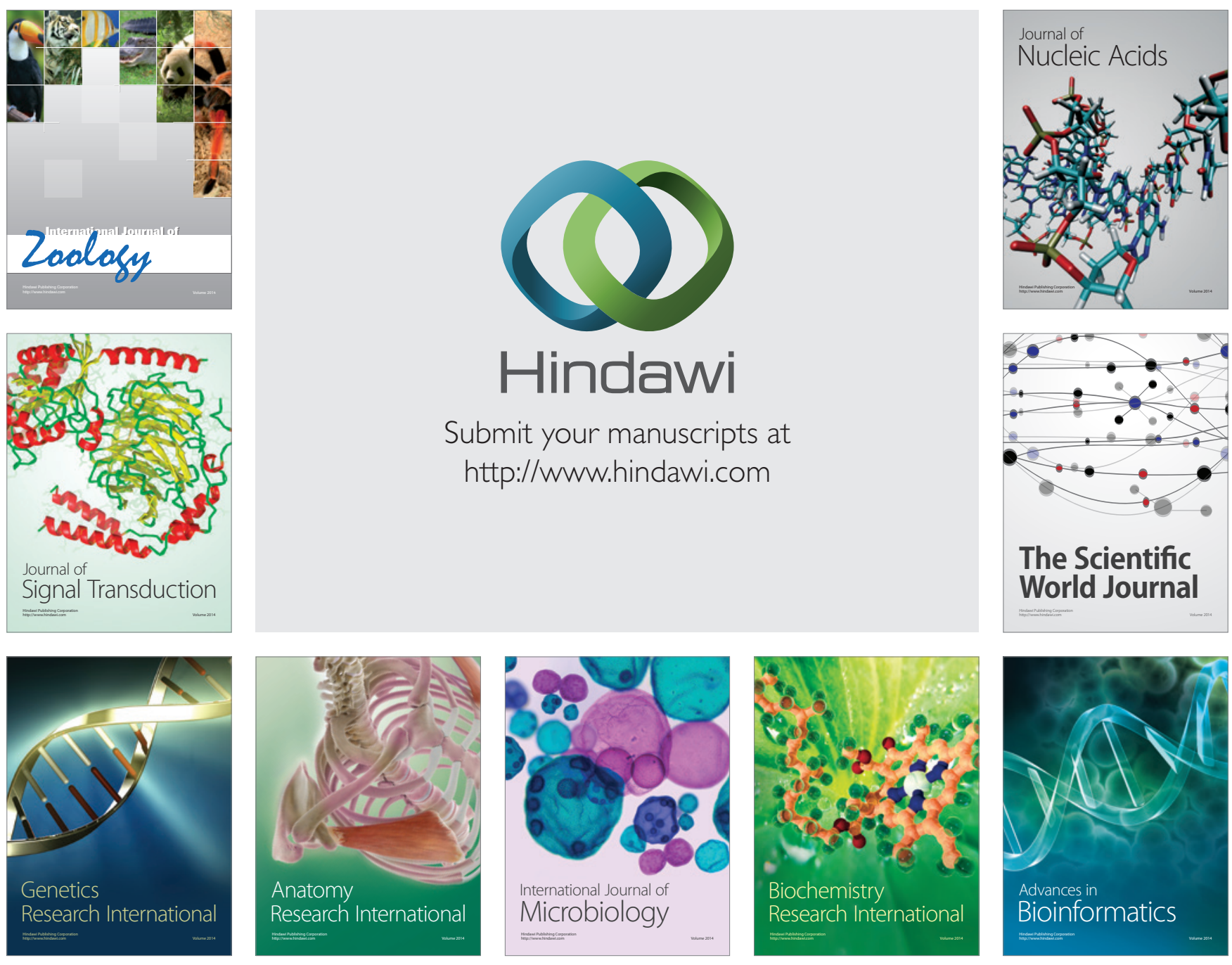

The Scientific World Journal
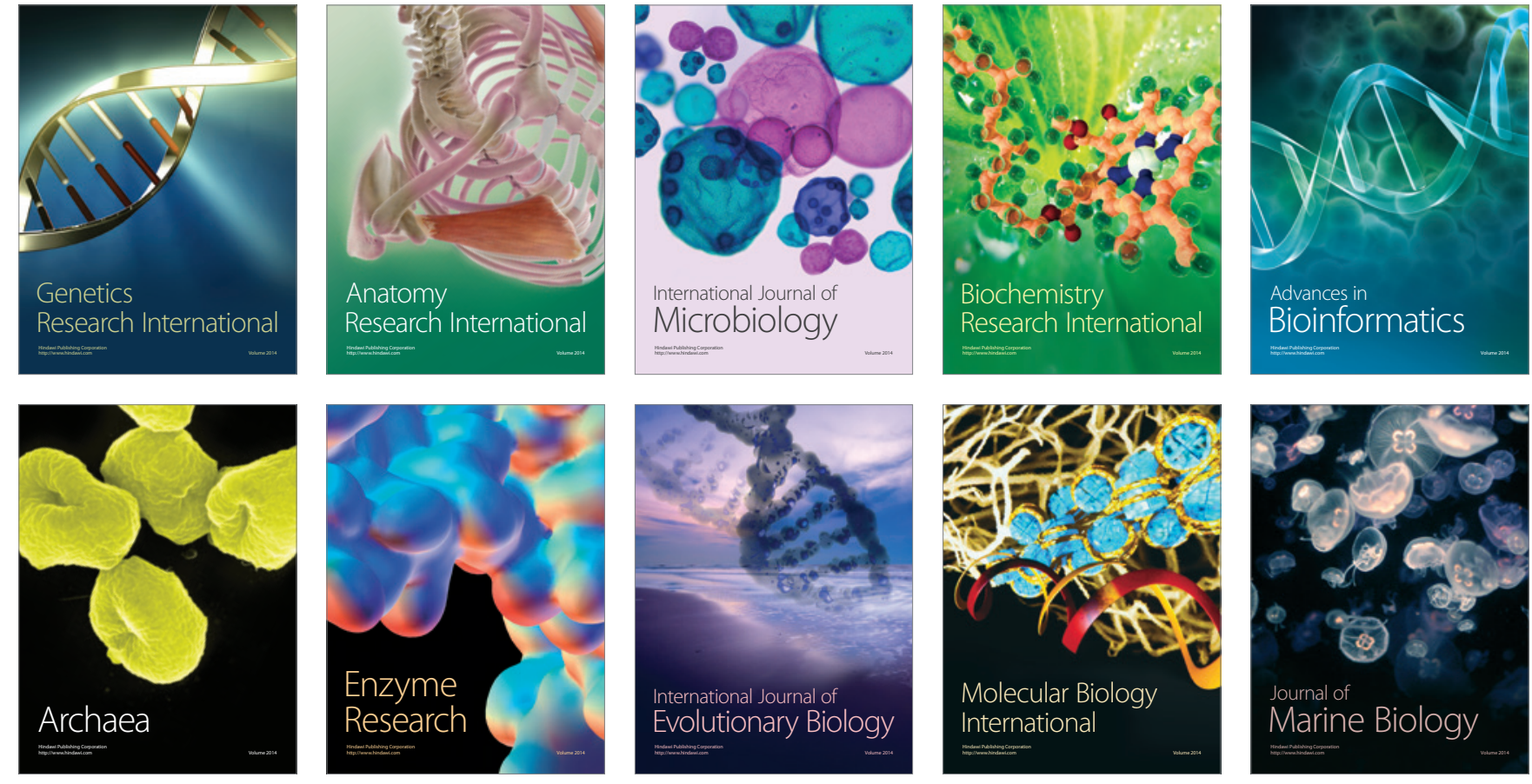